\title{
A New Robust Estımator for Value at Risk
}

\author{
Nuri Celik ${ }^{\mathrm{a} 1}$, Cihan Dincer ${ }^{\mathrm{b}}$ \\ ${ }^{a}$ Bartin University, Department of Statistics, 74100 Merkez, Bartin,Turkey \\ ${ }^{\mathrm{b}}$ Cologne Business School, Department of International Business, 50677, Cologne, Germany
}

\begin{abstract}
We consider a common risk measuring method namely Value-at-Risk (VaR). The easiest and the most prevalent method of calculating VaR is the variance-covariance method. This method is based on normal distribution assumption. However, there are a lot of inferences in literature that non-normal distributions are much more common than the normal distribution. Because of economic growth and political and financial issues, there can be possible higher or lower prices than normal ones in economic data, which are named outlier in statistic theory. In order to handle these data anomalies and distribution differences, robust estimation and testing methods have been determined and studied for last decades. In this study, we propose a new robust variancecovariance estimator for calculating VaR value of a given portfolio. Simulation results show that the proposed estimator is more robust than the corresponding normal theory solutions. Also, a real data for different economical markets are analyzed to show the performances of the proposed estimators.
\end{abstract}

Keywords: Value at Risk, Variance-Covariance Method, Robust Statistics, Outlier

\section{INTRODUCTION}

Estimating a loss in assets will not only contribute to financial businesses but also to other companies and individuals. Businesses, operating globally undertake more risk than those running their business on a national level. This additional threat needs to have an efficient risk management plan or otherwise the businesses will face financial problems or even bankruptcy. Understanding this threat international companies have started to develop countermeasures in order to asses and to defeat the risk.

Value at risk (VaR) is a method, recommended by Basel standards, which is applied by several financial businesses to measure the risk. It identifies the highest probability of loss of value in assets or a given portfolio in a given time period. In other words, it displays the highest possible loss in value held within a certain period of time and a given confidence interval. Researches have shown that businesses can protect themselves from risk if an accurate estimation of $\mathrm{VaR}$ is done previously. This will also ensure a company's sustainability. Due to this, many parties including governments, external auditors, businesses within a supply chain, and labor unions have a high interest in these information. Calculating VaR is widespread not only because of its easiness to compute, also due to the acceptance by several businesses. Furthermore, it is recommended by the Bank of International Settlement (BIS). The risk of a portfolio is illustrated by a one digit number, which appears convenient to financial entities.

There are several methods to calculate VaR, for instance the variance-covariance method (Jordan and Mackay, 1997), Exponentially Weighted Moving Average (EWMA) method (Hendricks, 1996), Historical Simulation and Monte Carlo simulation method (Holton, 1998), Extreme Values method (Longin, 2000 and Ho et. all. 2000), Kernel density method (Butler and Schacter, 1997) Generalized Auto Regressive Conditional Heteroscedasticity (GARCH) method (Alexander, 1996) and Fractionally Integrated ARCH (Beltratti and Maronna, 1999).

The simplest and the most common method is the variance-covariance method. This method is based on the assumption of normality. However, in literature, there are several studies underlining that non-normal distributions are more prevalent than the normal distribution in practice, see for example, Pearson (1932), Geary (1947), Huber (1981) and Tan and Tiku (1999). In addition, observations in a sample which are too small or too large as compared to the bulk of observations are called outliers. Since their presence adversely affects the efficiency of most statistical procedures (Tiku and Akkaya, 2004). Therefore, nonparametric methods and semi parametric methods have been studied to handle these distribution differences and data anomalies in order to calculate VaR values, see Duffie and Pan (1997), Pritsker (1997) and Kuester et all. (2006). However, using robust methods for analyzing in such situations are much more reliable than semi parametric and nonparametric methods. An estimator is called robust if

${ }^{1}$ Corresponding Author: ncelik@bartin.edu.tr 
it is fully efficient under the assumed model and maintains high efficiency under the plausible alternatives of the assumed model, see Tiku and Akkaya (2004). There are only a few studies predicting VaR values in a robust way in literature. The main focus of these studies is to estimation of tails of profit and loss distributions, see Mancini and Trojani, 2007 and Gebizlioglu et. all, 2011. Additionally, there is no previous work of estimating robust version of volatility. For this reason, we propose a new robust method for calculating VaR using robust variance-covariance matrix.

The rest of the paper organizes as follows, in chapter 2, we give a brief information about the standard variancecovariance method, then we define a robust method for estimating variance-covariance matrix and with this new matrix we calculate new robust VaR value for a given asset or portfolio. In the next chapter, we simulate data from various distributions used in statistical analysis and compare two estimators. Lastly, we apply this estimator for different real data examples. Conclusion is given at the end of the paper.

\section{VARIANCE-COVARIANCE METHOD}

This parametric approach identifies parameters, which influence the value of a portfolio. It also illustrates the highest possible loss which occur due to fluctuations on a certain probability level. It is the method used by the RiskMetrics methodology and developed by JP Morgan. The method is based on the assumption of normal distribution. By assuming the normal distribution $\mathrm{VaR}$ of portfolios can be calculated in terms of a linear function of standard deviation of assets.

With the variance-covariance method primarily the mean and the volatility (standard deviation) have to be attained in order to calculate VaR of portfolio, which contains $n$ financial assets. Afterwards, the weight vector $(w)$ has to be determined. Based on this, the mean and the standard deviation of the portfolio can be obtained

$$
\begin{aligned}
& \mu_{p}=\sum_{i=1}^{n} w_{i} \mu_{i}, \quad i=1,2, \ldots, N \\
& \text { And } \sigma_{p}=\sqrt{\sum_{i=1}^{N} \sum_{j=1}^{N} w_{i} w_{j} \sigma_{i j}} \quad i=1,2, \ldots, N ; j=1,2, \ldots, N
\end{aligned}
$$

respectively. where $\mu_{i}$ is the mean of ith asset, $w_{i}$ is the weight of $i$ th asset in portfolio, $\sigma_{i j}$ is the covariance between ith and $j$ th asset and $N$ is the number of assets in a given portfolio. Then VaR is calculated with the multiplication of this standard deviation and required probability, which is constant in normal distribution ( 1.96 with the probability \%95, 2.58 with the probability \%99.)

The advantage of this method is the easiness of computing. On the other hand, the disadvantage is the assumption of normal distribution. As illustrated in introduction part, using robust methods is more reliable in data analysis in the presence of outliers and distribution differences. The variance of a sample is not robust and affected by even one outlier. Therefore, VaR value based on the variance-covariance method is not robust as well. For this reason, we determine a robust estimator of variance-covariance matrix and the method for calculating VaR based on this robust variance-covariance matrix.

\section{ORTHOGONALIZED GNANADESIKAN-KETTERNRING ESTIMATE}

The Orthogonalized Gnanadesikan-Ketternring (OGK) estimator is based on the robust covariance matrix estimate defined by Gnanadesikan and Ketternring (1972). The defined variance-covariance matrix is symmetric but not necessarily positive semi definite. To overcome the problem of semi definiteness, Maronna and Zamar (2002) proposed a new estimate for mean vector and covariance matrices. The distinctive feature of the OGK estimation is that it combines the use of the actual measurements with an existing estimate of the covariance and hence implicitly accounts for any correlation between the sources of the measurements and for the way that these were obtained (Sequeira et all, 2011). The estimation procedure is very fast and easy to compute.

Let $x_{1}, x_{2}, \ldots, x_{n} \in \mathfrak{R}^{p}$ be a dataset and $\sigma($.$) and \mu($.$) be robust univariate dispersion and location statistics and$ Let $v(.,$.$) be a robust estimate of the covariance of two random variables. Maronna and Zamar(2002) define a new$ robust variance-covariance matrix $\mathbf{Z}(\mathbf{X})$ and mean vector $\mathbf{m}(\mathbf{x})$ as follows: 
1. Let $\mathbf{D}=\operatorname{diag}\left(\sigma\left(X_{1}\right), \ldots, \sigma\left(X_{n}\right)\right)$ and $\mathbf{y}_{\mathbf{i}}=\mathbf{D}^{-1} \mathbf{x}_{\mathbf{i}}, i=1,2, \ldots, n$

2. Compute the correlation matrix $\mathbf{C}=\left[C_{j k}\right]$, applying $v$ to the columns of $\mathbf{Y}$, that is $C_{j j}=1$, and $C_{j k}=v\left(Y_{j}, Y_{k}\right), j \neq k$.

3. Compute the eigenvalues $\lambda_{j}$ and eigenvectors $\mathbf{e}_{\mathbf{j}}$ of $\mathbf{C}$ and call $\mathbf{E}$ the matrix whose columns are the $\mathbf{e}_{\mathbf{j}}$ 's, so that $\mathbf{C}=\mathbf{E} \mathbf{\Lambda} \mathbf{E}^{\prime}$, where $\boldsymbol{\Lambda}=\operatorname{diag}\left(\lambda_{1}, \ldots, \lambda_{p}\right)$.

4. Let $\mathbf{A}=\mathbf{D E}$, and $\mathbf{z}_{\mathbf{i}}=\mathbf{E}^{\prime} \mathbf{y}_{\mathbf{i}}=\mathbf{A}^{-1} \mathbf{x}_{\mathbf{i}}$, so that $\mathbf{x}_{\mathbf{i}}=\mathbf{A} \mathbf{z}_{\mathbf{i}}$ and define $\mathbf{Z}(\mathbf{X})=\mathbf{A} \Gamma \mathbf{A}^{\prime}$ and $\mathbf{m}(\mathbf{x})=\mathbf{A} \mu$

where $\Gamma=\operatorname{diag}\left(\operatorname{var}\left(Z_{1}\right), \ldots, \operatorname{var}\left(Z_{p}\right)\right)$ and $\mu=\left(\mu\left(Z_{1}\right), \ldots, \mu\left(Z_{p}\right)\right)^{\prime}$.

Maronna and Zamar(2002) take $v$ the Gnanadesikan-Kettenring estimator (Gnanadesikan and Kettenring,1972) which is,

$$
U_{j k}=\frac{1}{4}\left[\sigma\left(Y_{J}+Y_{k}\right)^{2}-\sigma\left(Y_{J}-Y_{k}\right)^{2}\right], j \neq k
$$

Then the resulting estimate is called an OGK estimate. The procedure can be extended by iteration and by reweighting algorithm. To determine $\sigma($.$) and \mu($.$) and the properties of OGK matrix (consistent, semi definite,$ affine-equivariant) see Maronna and Zamar, (2002). For the influence function and other robustness properties of Gnanadesikan-Kettenring estimator also see Genton and Ma, (1999).

With this predefined robust estimator of variance-covariance matrix, we calculate VaR value of a portfolio with traditional calculation method. The only difference between standard variance-covariance method and this proposed method is the estimator of volatility, which contains robustness and efficiency.

\section{Simulation STUdY}

In this section, we compare variance-covariance method which is traditionally used with the proposed robust method in terms of VaR values. All the simulation results are based on [100,000/n] Monte Carlo runs. We use some models, which distributed near normal or contains outliers namely Dixon's outlier model $-(n-1)$ observation come from normal distribution and one outlier (not known which one) comes from normal distribution with higher standard deviation value than the distribution that the bulks of data come from-, Contamination model - $(1-p) \%$ of the observation come from normal distribution with standard deviation $S$ and $p \%$ of the observation come from normal distribution with standard deviation not equal to $S$, where $p$ is a proportion differs from [0,1]- and Mixture model (1-p)\% of the observation come from normal distribution with standard deviation $S$ and $p \%$ of the observation come from other distributions near normal-. We use the following sample models to represent a large number of plausible alternatives.

Sample Models:

Model (1): Dixon's outlier model: $(n-1)$ observations come from $N(0,0.01)$ but one observation (we do not know which one) comes from $N(0,0.04)$

Model (2): Dixon's outlier model: $(n-1)$ observations come from $N(0,0.01)$ but one observation (we do not know which one) comes from $N(0,0.1)$

Model (3): Contamination model: $0.90 N(0,0.01)+0.10 N(0,0.03)$

Model (4): Contamination model: $0.90 N(0,0.01)+0.10 N(0,0.05)$

Model (5): Mixture model: $0.90 N(0,0.01)+0.10$ Student $t(2)$

Model (6): Mixture model: $0.90 N(0,0.01)+0.10$ Student $t(7)$ 
American Research Journal of Business and Management, Volume 1, Issue 1, February 2015

ISSN 2379-1047

Simulation results are given in Table 1.

Table1 Simulated VaR values of different distributions

\begin{tabular}{|c|c|c|}
\hline Distribution & Var-Cov Method & OGK Method \\
\hline Model (1) & 0.0114 & 0.0106 \\
\hline Model (2) & 0.0135 & 0.0103 \\
\hline Model (3) & 0.0133 & 0.0109 \\
\hline Model (4) & 0.0182 & 0.0114 \\
\hline Model (5) & 0.0231 & 0.0115 \\
\hline Model (6) & 0.0201 & 0.0108 \\
\hline
\end{tabular}

Table 1 shows that, VaR values calculated with OGK method are smaller than the values calculated with traditional method. In addition, the standart deviations, estimated with robust method are much more efficient than traditionally estimated standart deviations. This outcome approves the expectation since it is within the nature of robust statistics.

\section{APPLICATION}

We analyzed several different real data from different financial markets. We investigated Dow Jones from United States of America, DAX from Germany, Nikkei from Japan and BIST from Turkey. For obtaining a good portfolio, we took 5 different assets from each stock exchange within the years 01.01.2013 to 31.12.2013. Two operating in the financial market, one in the fitness sector, one in aviation and one in information technology. It may be noted that for the application part the weight matrix $w$ is $[0.2 ; 0.2 ; 0.2 ; 0.2 ; 0.2]$. In other words the proportion of different assets in portfolio is equal. After obtaining the data from different stock exchanges, the descriptive statistics are calculated. Then the Q-Q plots are drawn for determining the distribution. The data are transformed into the form $\frac{P_{t}-P_{t-1}}{P_{t-1}}$, where $P_{t}$ is the closing price for a given asset in certain time $t$.

The descriptive statistics of the assets of the German market can be found in Table 2. Respectively, these descriptive statistics of the Japanese, US American and Turkish stock exchange are given in Table 3,4 and 5.

Table2. Descriptive statistics of assets from DAX

\begin{tabular}{|l|c|c|c|c|}
\hline & Mean & Standard Deviation & Skewness & Kurtosis \\
\hline Asset 1 & -0.0009 & 0.0168 & 0.295 & 2.834 \\
\hline Asset 2 & 0.0004 & 0.0191 & -0.120 & 0.871 \\
\hline Asset 3 & 0.0008 & 0.0209 & 0.174 & 1.945 \\
\hline Asset 4 & -0.0011 & 0.0171 & 0.680 & 0.985 \\
\hline Asset 5 & -0.0021 & 0.0185 & -0.045 & 1.068 \\
\hline
\end{tabular}

Table3. Descriptive statistics of assets from Nikkei

\begin{tabular}{|l|c|c|c|c|}
\hline & Mean & Standard Deviation & Skewness & Kurtosis \\
\hline Asset 1 & -0.001 & 0.0164 & -0.371 & 1.252 \\
\hline Asset 2 & 0.0008 & 0.0561 & 0.437 & 2.188 \\
\hline Asset 3 & -0.0002 & 0.0233 & 0.150 & 0.353 \\
\hline Asset 4 & -0.0008 & 0.0158 & 0.172 & 0.426 \\
\hline Asset 5 & -0.0007 & 0.0168 & -0.343 & 2.472 \\
\hline
\end{tabular}

Table4. Descriptive statistics of assets from Dow Jones

\begin{tabular}{|c|c|c|c|c|}
\hline & Mean & Standard Deviation & Skewness & Kurtosis \\
\hline Asset 1 & -0.0008 & 0.0104 & 0.169 & 1.955 \\
\hline Asset 2 & -0.0001 & 0.0183 & 0.179 & 1.638 \\
\hline Asset 3 & 0.0008 & 0.0168 & 0.211 & 1.295 \\
\hline Asset 4 & -0.0007 & 0.0183 & -0.118 & 0.950 \\
\hline Asset 5 & -0.0028 & 0.0209 & 0.465 & 1.968 \\
\hline
\end{tabular}


American Research Journal of Business and Management, Volume 1, Issue 1, February 2015

ISSN 2379-1047

Table5. Descriptive statistics of assets from BIST

\begin{tabular}{|c|c|c|c|c|}
\hline & Mean & Standard Deviation & Skewness & Kurtosis \\
\hline Asset 1 & 0.0022 & 0.0249 & 0.082 & 0.285 \\
\hline Asset 2 & 0.0045 & 0.0342 & 0.533 & 1.855 \\
\hline Asset 3 & 0.0029 & 0.0231 & -0.384 & 1.853 \\
\hline Asset 4 & 0.0059 & 0.0406 & 1.048 & 2.339 \\
\hline Asset 5 & 0.0012 & 0.0251 & 0.210 & 1.902 \\
\hline
\end{tabular}

In order to determine the distribution of each assets, we use Q-Q plot technique. The Q-Q plots of each assets from different stock exchange are given in Figure 1-4. As illustrated in Figure 1, the Q-Q plots of asset 1,2,3 and 5 have some outliers, on the other hand, the distribution of asset 4 is not exactly normal but near normal. In Figure 2,3 and 4 we have also some outliers and divergence from normal distribution.
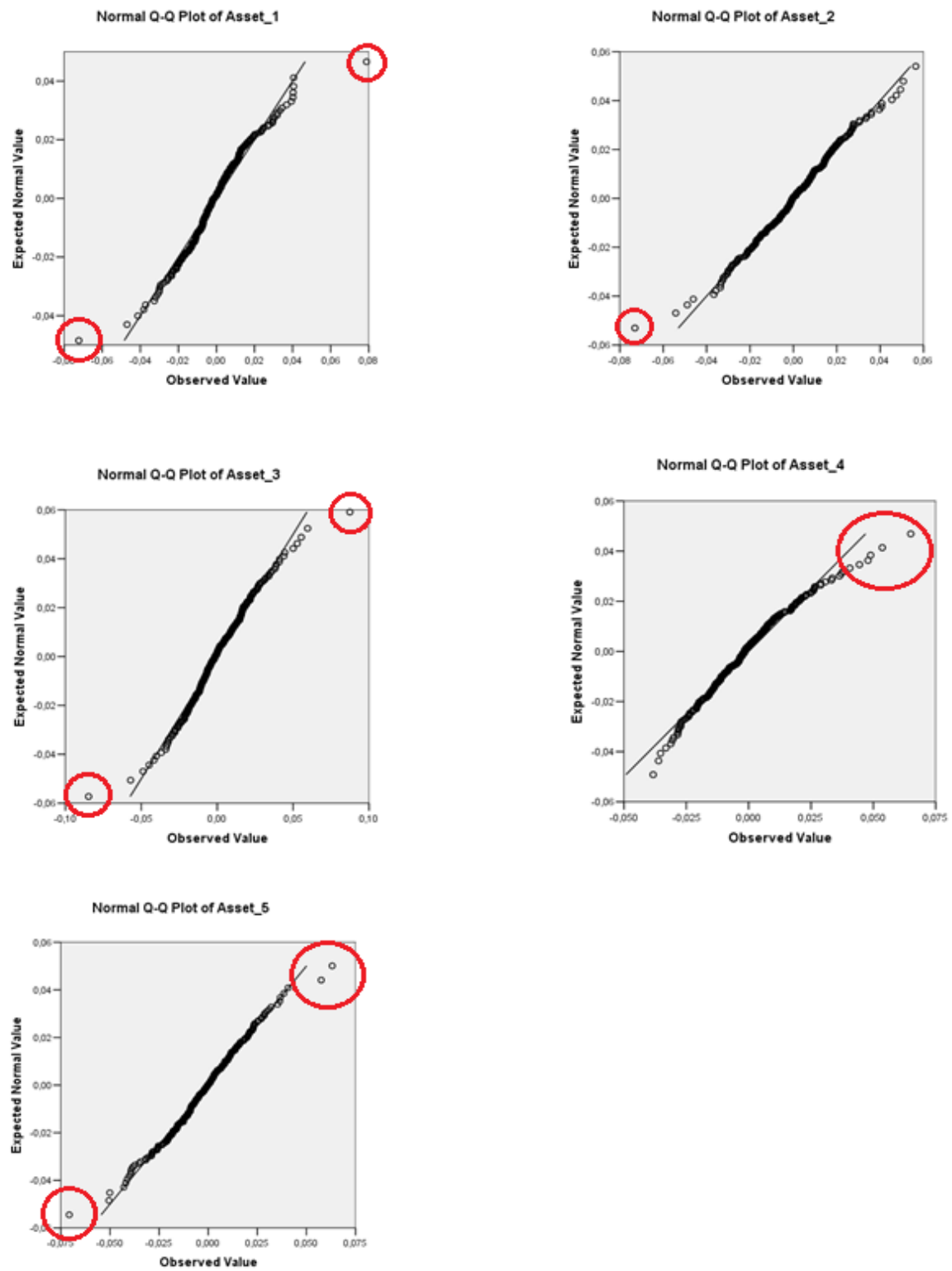

Figure1. $Q-Q$ plots of the assets from $D A X$ 
American Research Journal of Business and Management, Volume 1, Issue 1, February 2015 ISSN 2379-1047
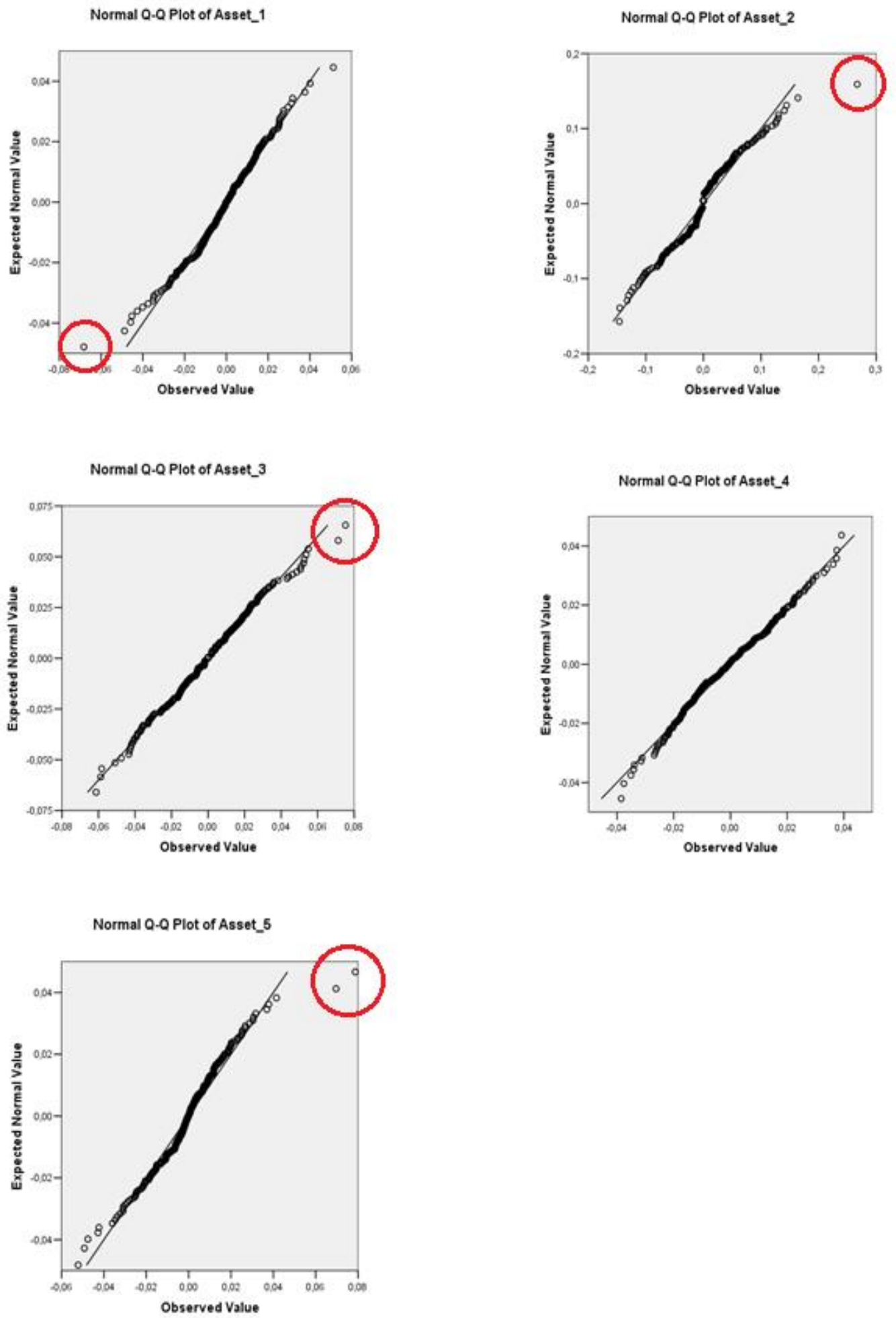

Figure2. $Q-Q$ plots of the assets from Nikkei 
American Research Journal of Business and Management, Volume 1, Issue 1, February 2015 ISSN 2379-1047
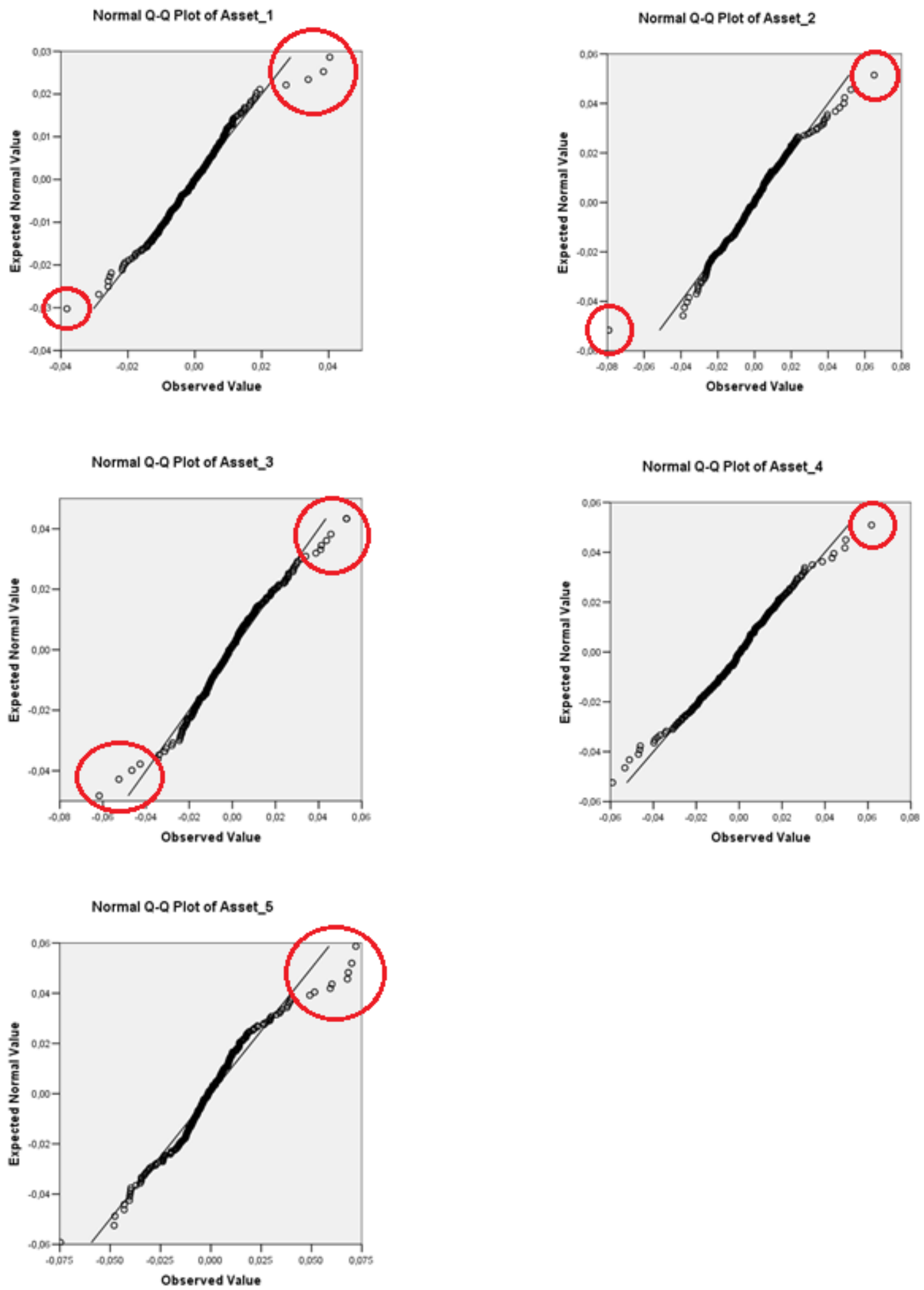

Figure3. $Q-Q$ plots of the assets from Dow Jones 

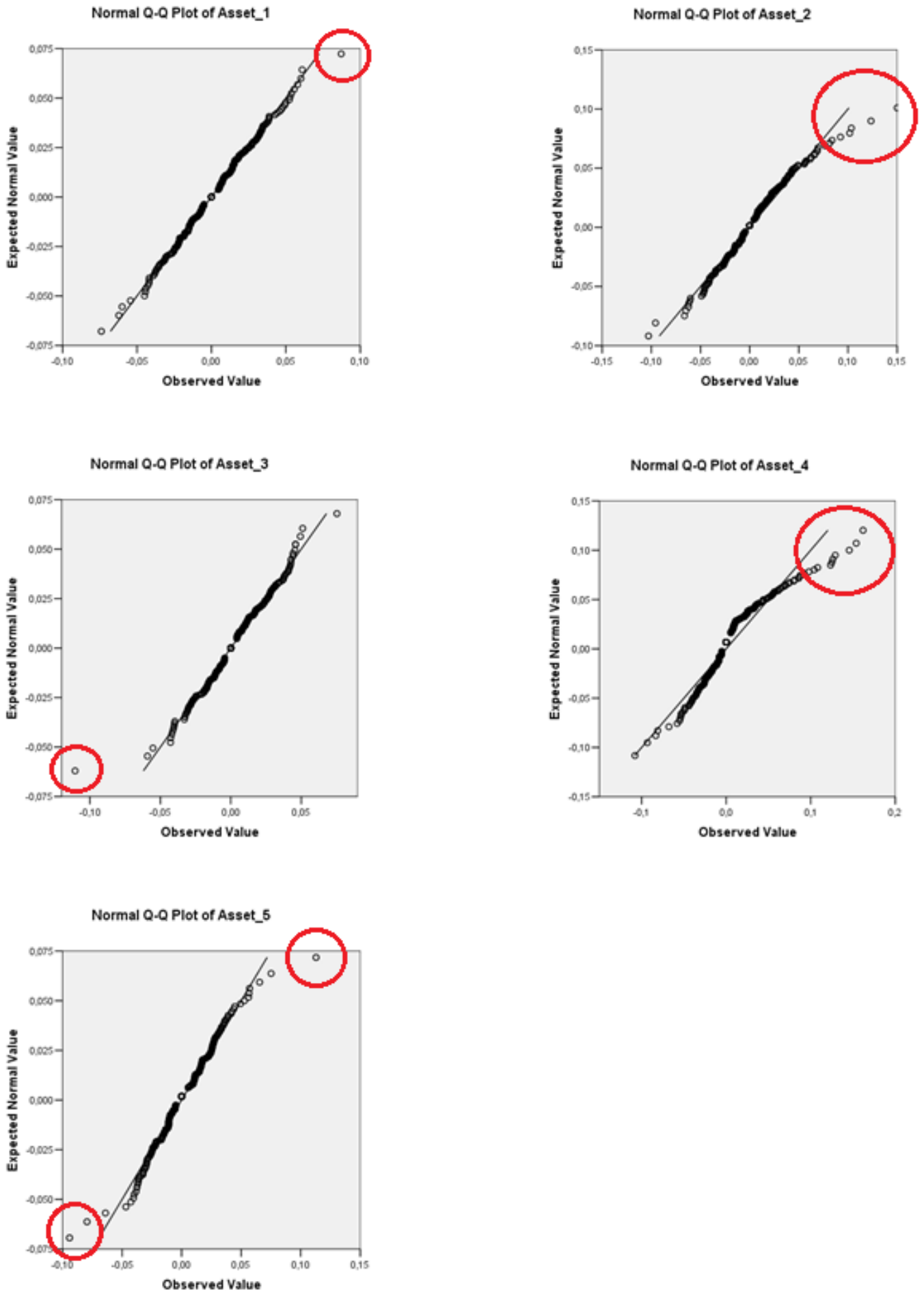

Figure4. $Q-Q$ plots of the assets from BIST

To conclude, we give VaR values of different portfolios from each stock exchanges in Table 6. VaR values are calculated with $\% 95$ confidence levels, for one week and for 1000 dollars. As Table 6 shows, VaR values calculated with the traditional variance-covariance method are higher than VaR values calculated with the robust method. 
Therefore, robust method is not affected by outliers and distribution divergences. The outcome of this method is more reliable and more accurate.

Table6. VaR values of different stock exchanges

\begin{tabular}{|c|c|c|}
\hline Stock Exchange & Var-Cov Method & OGK Method \\
\hline Germany & 10.973 & 4.531 \\
\hline Japan & 20.536 & 13.162 \\
\hline USA & 9.001 & 1.552 \\
\hline Turkey & 18.910 & 14.468 \\
\hline
\end{tabular}

\section{CONCLUSION}

Traditionally, variance-covariance method are used in the context of Value at Risk calculation. The method is applied with the normal distribution assumption. However, efficiencies of the standard deviation estimator are low when the normality assumption is not satisfied. Also, the sample standard deviation estimator is nonrobust when the distribution is not normal and outliers in a sample are existing.

In this paper, we define a new method of calculating VaR value with robust variance-covariance matrix, since the robust methods have been used to handle these data anomalies and departures from normality. Simulation studies and real data analysis underline that the VaR values calculated with robust method is more reliable and more accurate than those calculated with variance-covariance method. Additionally, variance-covariance matrices estimated with robust method are more efficient and more robust than the matrices estimated with normal theory.

\section{REFERENCES}

[1] Alexander, C. (1996), "Volatility and Correlation: Measurement, models and applications", Risk Management and Analysis, John Wiley and Sons.

[2] Beltratti, A. and Maronna, C. (1999), "Computing value at risk with high frequency data", Journal of Emprical Finance, 6, 431-455.

[3] Butler, J.S. and Schachter, B. (1997), "Estimating Value-at-risk with a precision measure by combining Kernel estimation with historical simulation", Review of Derivative Research, 1, 371-390.

[4] Duffie, D. and Pan, J. (1997), "An overview of Value at risk", Journal of Derivatives, 4, 7-49.

[5] Geary, R.C. (1947), “Testing for normality”, Biometrika, 34, 209-242.

[6] Gebizlioglu, O. Senoglu, B. and Kantar, Y.M. (2011), "Comparison of certain value-at-risk estimation methods for the two-parameter Weibull loss distribution", Journal of Computational and Applied Mathematics, 235, 3304-3314.

[7] Genton, M.G. and Ma, Y. (1999), "Robustness properties of dispersion estimators" Statistics and Probability Letters, 44, 343-350.

[8] Gnanadesikan, R. and Kettenring, J.R. (1972), "Robust estimates, residuals and outlier detection with multiresponse data.", Biometrics, 28-1, 81-124.

[9] Hendricks, D. (1996), "Evaluation of Value-at-Risk models using historical data", FRBNY Economic Policy Rewiew, 2, 39-69.

[10] Ho, L.C., Burridge, P., Caddle, J. and Theobald, M. (2000), "Value-at-Risk applying the extreme value approach to Asian markets in the recent financial turmoil", Pacific-Basin Finance Journal, 8, 249-275.

[11] Holton, A.G. (1998), "Simulating Value-at-Risk", Risk, 11-5, 60-63.

[12] Huber, P.J., (1981), "Robust Statistics", Jonh Wiley, New York

[13] Jordan, J.V. and Mackay, R.J. (1997), "Assesing Value at Risk for equity portfolios: Implementing alternative techniques", Derivatives Handbook Risk Management and Control, John Wiley and Sons.

[14] Kuester, K., Mittnik, S. and Paolella, M.S. (2006), "Value at Risk nprediction: A comparison of alternative strategies", Journal of Financial Econometrics, 4, 53-89.

[15] Longin, F.M. (2000), "From value at risk to stress testing: The extreme value approach", Journal of Banking and Finance, $24,1097-1130$.

[16] Mancini, L. and Trojani, F. (2010), "Robust Value at Risk Prediction”, Journal of Financial Econometrics, 9, 281-313.

[17] Maronna, R.A. and Zamar, R.H. (2002), "Robust estimates of location and dispersion for high-dimensional datasets", Technometrics, 44-4, 307-317.

[18] Pearson, E.S. (1932), “The analysis of variance in cases of nonnormal variation”, Biometrika, 23, 114.133. 
American Research Journal of Business and Management, Volume 1, Issue 1, February 2015

ISSN 2379-1047

[19] Pritsker, M. (1997), "Towards, assesing the magnitude of Value at Risk errors due to the errors in the correlation matrix", Financial Engineering News, 2, 14-16.

[20] Sequeira, J., Tsourdos, A., and Lazarus, S.B. (2011), "Robust Covariance Estimation for Data Fusion From Multiple Sensors", Instrumentation and Measurement, IEEE Transactions on, 12, 3833-3844.

[21] Tan, W.Y. and Tiku, M.L. "Sampling distributions in terms of Laguerre Polynomials with applications", New Age International (formerly, Wiley Eastern), New Delhi, 1999.

[22] Tiku, M.L. and Akkaya, A.D. Robust Estimation and Hypothesis Testing”, New Age International (P) Limited, Publishers (2004), New Delhi, 337pp. 